\title{
Intrusion Detection Using Growing Hierarchical Self-Organizing Maps and Comparison with other Intrusion Detection Techniques
}

\author{
Aleksei Kharitonov \\ Aalen University \\ Aalen, Germany \\ aleksei.kharitonov@hs-aalen.de
}

\author{
Axel Zimmermann \\ Aalen University \\ Aalen, Germany \\ axel.zimmermann@hs-aalen.de
}

\begin{abstract}
Intrusion detection systems (IDS) based on machine learning (ML) can be used to detect anomalies in data traffic. Common challenges for IDSs are low detection rates, high false alarm rates, and the need to process large amount of data. In order to overcome these challenges various types of supervised, semi-supervised and unsupervised ML methods are being widely researched. However, the need for high-performance processing capabilities in order to perform the calculations restricts the use in industrial control systems (ICS) with usually small embedded processing elements. In this paper, a comparison of key ML methods is carried out that focuses on data traffic and processing requirements for the application to ICS. Furthermore, a variant of growing hierarchical self-organizing maps (GHSOM) is described that proposes a new method to detect anomalies based on the GHSOM behaviour. The influence of the GHSOM parameters on the detection ratio of different kinds of attacks on a production line for the proposed method is examined. To detect attacks on the ICS, growth criteria of the GHSOM have been applied as alarm generating conditions. The comparison of the performance of GHSOM, Feedforward Artificial Neural Network (ANN), Support Vector Machine (SVM) and Local Outlier Factor (LOF) took place based on receiver operating characteristics of the classification between normal and anomalous data captured from a real industrial line testbed. The proposed method has shown comparable classification results with one-class SVM and LOF as novelty detection method in the studied case scenario.
\end{abstract}

\section{KEYWORDS}

self-organizing maps; GHSOM; intrusion detection; industrial network security

\section{ACM Reference Format:}

Aleksei Kharitonov and Axel Zimmermann. 2019. Intrusion Detection Using Growing Hierarchical Self-Organizing Maps and Comparison with other Intrusion Detection Techniques. In 5th ACM Cyber-Physical System Security Workshop (CPSS '19), July 8, 2019, Auckland, New Zealand. ACM, New York, NY, USA, 11 pages. https://doi.org/10.1145/3327961.3329531

Permission to make digital or hard copies of part or all of this work for personal or classroom use is granted without fee provided that copies are not made or distributed for profit or commercial advantage and that copies bear this notice and the full citation on the first page. Copyrights for third-party components of this work must be honored.

For all other uses, contact the owner/author(s).

CPSS '19, July 8, 2019, Auckland, New Zealand

(C) 2019 Copyright held by the owner/author(s).

ACM ISBN 978-1-4503-6787-5/19/07.

https://doi.org/10.1145/3327961.3329531

\section{INTRODUCTION}

Motivation. The modern industry is going to become more integrated to the internet. Lots of safety and security questions have to be taken into consideration. Even the isolation of industrial systems from other environments through air-gap can not guarantee a secure system. Prominent examples of attacks on industrial control systems (ICS) are the Stuxnet worm that damaged Iranian nuclear centrifuges or the Shamoon virus that attacked Saudi oil companies. Therefore, ICS have to be protected. One of the possibilities to secure an industrial plant is an intrusion detection system (IDS) that observes data traffic in the industrial process, like for example via self-organizing maps (SOM). There are many different architectures for intrusion detection using self-organizing maps and its successors [4]. Many IDS were evaluated on datasets such as KDDCup'99 and KDD-NSL, where normal vectors and vectors derived from different types of attacks should be distinguished. But for building an online-capable intrusion detection system, especially for industrial systems a short reaction time should be provided. If an attacker sends a malicious payload in a single connection, then the prevention system based on connection-derived features can't react before the connection is established and the malicious payload is sent. To a achieve better reaction time for IDS, the analysis of single packages is desirable.

The reason why GHSOM was the method of choice in the present work is that many research groups achieved good results for the implementation of GHSOM in intrusion detection. A short overview of related works is given in the second section (background). In cases where the highest detection and lowest false alarm rates were achieved by using GHSOM on well-known datasets, the GHSOMs were chosen as a kernel of the proposed intrusion detection algorithm in an industrial line. In contrast to most of the other IDS evaluations that are based on publicly available connection-oriented datasets, a self-captured dataset based on the packet data in a real manufacturing line testbed was used. A property of SOM/GHSOM is the ability to group data into nodes and it thereby reduces the number of input vectors to a certain number of nodes. In the present work it was intended to use the nodes of the GHSOM as a normal model for the intrusion detection on an industrial line.

Intrusion detection in industrial lines. Because of the cyclic behaviour of industrial control systems, lots of the same or similar data occur frequently. This property can be used for mapping of all the network traffic for a single network participant onto its own data model. A further characteristic of a production line is its deterministic behaviour in the way of communication, i.e. participants in the data exchange usually stay the same. The difference between the 
anomalous data and normal data can be expressed as a dissimilarity measure of describing features for both of them.

For intrusion detection in industrial networks, the relevant data have to be collected and then analysed. There are two methods to get the needed data for the intrusion detection [15]. The first is to obtain data passively and the other one is to periodically actively acquire data from a device, e.g. programming logic controller (PLC). The active interconnection of an IDS with the industrial network can get more data including status and state messages from the target to detect sophisticated protocol attacks [11]. But active polling is not always possible. It also can cause a CPU overload and create a new security vulnerability by providing itself as an attack platform for denial-of-service attacks. To reduce CPU usage and to implement an IDS in the target itself, a scheduled IDS can be implemented [1]. But the integrated IDS is not always applicable also. Besides that, the status polling interface should be secured, too. Moreover, an active information polling has to be adapted for every system that has to be secured by IDS. The passive method monitors only the data that go over the industrial line. This method needs a non-invasive data observation mechanism only, which can be easier integrated into the industrial networks, e.g. a method using port mirroring of the Ethernet switches [15]. Research on industrial intrusion detection systems was carried out by Jardine et al. and Hadžiosmanović et al., among others [11, 15]. Jardine et al. used an active monitoring of the PLC [15]. Hadžiosmanović et al. tried to monitor variables in the PLC and detect deviant behaviour [11] Ghaeini et al. have proposed a hierarchical monitoring intrusion detection system (HAMIDS) for ICS which is based on Bro IDS with extensions, e.g. Ethernet/IP and common industrial protocol (CIP) dissectors [9]. The authors test payload data to detect networkbased malicious traffic. The in-depth analysis could detect focused attacks on industrial applications. Inoue et al. have compared the applicability of deep artificial neural networks adapted to time series and one-class Support Vector Machine [12]. The data were generated by a cyber-physical system (CPS) from the Secure Water Treatment (SWaT) testbed [14]. While the one-class SVM approach provided a slightly better recall, the deep ANN approach has shown a better F score. Another work using the SWaT testbed was published by Kravchik and Shabtai [16]. An attack detection approach using 1D convolutional neural networks is proposed, whereat over $86 \%$ of all attacks were recognized using this method. An approach for intrusion detection using state analysis of the ICS was proposed by Ghaeini et al. [8].

\section{Contributions.}

- The main point of the work is to use the GHSOM as a tool to check its applicability for intrusion detection on a real industrial line testbed, which hasn't been investigated before and was successfully carried out.

- The two growth conditions of the algorithm were applied as anomaly detection conditions. If testing vectors would trigger a growth of the map, they will be assumed to be anomalous.

- The used GHSOM parameters don't only contribute to the geometry of the map itself, but also strongly influence the proposed anomaly detection algorithm.
- The proposed method of GHSOM was superior over the supervised training method using ANN in the case when certain attack vectors weren't trained in the model and afterwards incorrectly classified by the ANN. The GHSOM has also shown better results in the case of semi-supervised training in comparison to one-class SVM.

\section{BACKGROUND}

The following section will give a short overview of approaches for IDS, methods based on anomaly detection and GHSOM for intrusion detection.

Approaches for IDS. Based on the observed data, important features have to be derived and have to be watched over the whole production time to achieve a fast reaction against cyber attacks. This function has to be implemented in an intrusion detection system. Commonly used algorithms for IDS are for example SOM, support vector machines (SVM), artificial neural networks (ANN), Bayesian Networks and the local outlier factor (LOF) method. Among the main approaches that are used for intrusion detection are the two following [6, 10, 17, 21]. The first one is a rule or signature-based approach where a set of undesired behaviours of a system has to be described. The second one is anomaly-based detection trying to build a normal model of the systems' data traffic, whereas deviant behaviour has to be marked as an anomaly and has to be investigated. The first approach could have better true positive rates or attack detection rates for known possible attacks, while at the same time being almost unable to detect attacks that were derived from already known ones due to its strictly predefined rules. The anomaly-based approach tries to overcome the restrictions of a rule-based detection and to detect cyber attacks that were unknown before. Among other detection techniques, academic research activity on anomaly-based detection techniques has grown. The number of academical publications about protection of field devices, PLCs and industrial communication protocols has increased. Being most researched, the machine learning-based detection has grown in the last few years [21]. Rubio et al. have analysed many different kinds of IDS architecture and attack detection methods [21].

Methods based on anomaly detection. There are many different approaches using anomaly-based intrusion detection. The following section will give a short overview of some common anomaly-based detection methods, namely multi-class and one-class detection, the neighbour-based and clustering-based approaches and SOM.

In case of multi-class classification, a dataset can contain many classes that are labelled and trained as a normal model occupying one or more points of the feature space. In the testing mode, anomalies are detected as outliers having a distance to normal vectors, i.e. exceeding the boundaries of a normal class.

The one-class anomaly detection exhibits only one normal class. Points in the feature space that don't fit to the normal class boundary are detected as anomaly [3]. This classification can be achieved for example using SVM. The main advantage of the classification is the object determination and fast testing phase. However, for multiple classes correct labels for all vectors should be provided, which is not always possible. 
Another approach to anomaly detection is the neighbour-based technique. The similarity between feature vectors is usually measured as a distance function, e.g. Euclidean or Manhattan distance. It is assumed that the anomalies occur far from their closest neighbours in the feature space, and the normal data tend to be grouped densely to each other. For each testing point an inverse of the data density in some surrounding or the distance to its neighbours can be used as a anomaly score. The k-nearest neighbours algorithm is the implementation of this technique. The algorithm is purely unsupervised and no labels are needed. Sometimes the algorithm fails if there are not enough close neighbours, which results in an increase of false negatives. Also this algorithm tends to produce a higher false positive rate if some vectors from the testing dataset have less similar vectors in the training dataset. The computational complexity in the testing phase is higher than in classification approach [3].

The clustering-based approach is another technique used in anomaly detection. It is mostly consisting of unsupervised learning and semi-supervised clustering processes. There are some different assumptions that are used to distinguish between normal and anomalous data. Assuming that either normal data build a cluster and anomalies don't, or that anomalies lie far away from the cluster's centroid and don't belong to any cluster, or alternatively to small or sparse clusters, different anomaly detection algorithms are used. The main advantages of this technique are its unsupervised training mode and fast testing phase, during which data are compared with a small number of clusters. The drawbacks are high computational costs and sensibility to the clustering algorithm. Some of the implementations are SOM, K-means clustering, etc. [3].

Although the SOMs are mostly used as a visualization technique for high-dimensional data onto a two-dimensional map, their ability to converge a given number of multidimensional points (nodes) to some data pattern enables the appliance for other purposes, too. The SOM places nodes at the average value of the input data mapped in the neighbourhood. The input vectors are weighted by the neighbourhood function. This method is attractive for the use in industrial intrusion detection systems. Training a SOM with random data results in a lattice (in case of $2 \mathrm{D}$ projection), which places the vertices (nodes) evenly around the feature map. Because of this, the method is practically unusable for highly variate data. Due to periodical behaviour of industrial control systems it is assumed that the SOM algorithm should be able to train a model with a small amount of nodes needed and that model can be used in intrusion detection by comparing the testing data with it. To overcome some restrictions of the SOM algorithm like static topology and inability to represent hierarchical dependency of data, a modification called growing self-organizing maps (GHSOM) has been developed by Rauber et al. [20]. Unlike the predecessor SOM, the growing hierarchical self-organizing maps (GHSOM) take also into account the hierarchical dependency of the input data. In addition to that, GHSOM adjust the architecture of the layers which could contain multiple growing SOMs.

Related works concerning GHSOM for intrusion detection. Different research groups have evaluated GHSOM and its modifications for the purpose of application in intrusion detection. The following works prove that by using GHSOM high detection rates can be obtained. However, those works like many others use provided datasets to test their algorithms. Ortiz et al. proposed a GHSOM with relabelling technique to solve the classification problem evaluated for 24 classes in the NSL-KDD dataset [18]. In this approach a majority of neighbours defines the node's label for the best matching unit (BMU). The authors claim that the detection of normal and anomalous patterns exceeds $99 \%$. Palomo et al. used the KDD Cup'99 dataset to evaluate their proposed intrusion detection system based on GHSOM [19]. After dividing the dataset into two smaller ones, a detection rate of at least $94.55 \%$ and a false positive rate of at most $2.37 \%$ was achieved. In the work of Ippoliti et al. multiple modifications of GHSOM were proposed [13]. An evaluation was carried out on the KDDCup'99 dataset. To improve the detection rate, a threshold-based training process was established, where every feature value is compared with the quantization error. Thereby it was attempted to detect anomalies in a single feature of the vector, whilst the distance of the vector to the best matching unit is smaller than the quantization error. The next modification implies a range-based normalization of features to reduce the influence of the small ranged values on the distance calculation. Another change in the algorithm is the adaptive adjustment of detection thresholds for each node. The overall accuracy for the proposed method was $93.53 \%$, compared to $92.21 \%$ for the unmodified GHSOM. Another work about intrusion detection based on GHSOM uses a mutual information coefficient for the KDD'99 dataset and clustered different attacks onto a hierarchical map [23].

\section{GROWING HIERARCHICAL SELF-ORGANIZING MAPS}

GHSOM like SOM project high-dimensional vectors onto a lowdimensional (usually 2D) map. An example of a GHSOM topology is shown on Figure 1. The shown map has three layers. The grey units possess an underlying map that provides better granularity of the vectors mapped on the unit.

It was proposed by Rauber et al. to overcome limitations of SOM, such as static map topology that is required to determine the size of the map before the training phase [20]. This condition makes the definition of the map size more difficult, because information about the input data is not yet available, as well as the possibility to graphically describe a hierarchical relationship of the input data. As stated by the authors, the visualization can aid in network forensics. In the research work of Palomo et al. an approach for visualization of network forensics traffic data was presented [19]. This approach intends to facilitate the graphical presentation of possible anomalies.

Training phase. In order to generate a data model based on GH$\mathrm{SOM}$, a training process has to take place. Every layer of the $\mathrm{GH}-$ SOM has one or several growing SOMs. Every single map has an overlaying node. This node for the first layer map is a single node in so-called layer 0 . Initially the GHSOM starts with a map containing $2 \times 2$ nodes. The training process for the GHSOM is similar to SOM with some additional steps. Assuming that the set $X$ is a training set and $V$ is a set of multidimensional vectors of the nodes in the map are given, then like in the case of SOM for a single given vector $x_{i} \in X$ and $v_{i} \in V$ the best matching unit or also called winner $c$ 


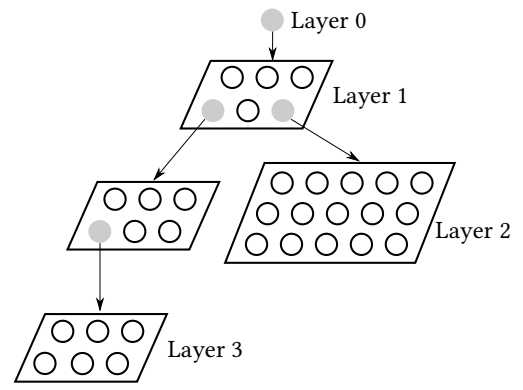

Figure 1: GHSOM structure example.

can be found by (1).

$$
c=\arg \min _{i}\left(\left\|x_{i}-v_{i}\right\|\right)
$$

The distance between a vector $x$ and a best matching unit $v_{c}$ is calculated by (2).

$$
d\left(x, v_{c}\right)=\left\|x_{i}-v_{c}\right\|
$$

The norm of (2) is often expressed as the Euclidean distance between two given vectors $x=\left(x_{1}, x_{2}, \ldots, x_{n}\right)$ and $v=\left(v_{1}, v_{2}, \ldots, v_{n}\right)$. The distance function could be denoted as $d(x, v)=\sqrt{\sum_{k=1}^{n}\left(x_{k}-v_{k}\right)^{2}}$.

Then the vectors of the winning node and its neighbours are updated according to (3). The adaptation function is denoted discretely. This means that $t$ represents a time in the training process usually assumed as a current iteration number.

$$
v_{i}(t+1)=v_{i}(t)+\alpha(t) \cdot h_{c i}(t) \cdot\left(x(t)-v_{i}(t)\right)
$$

Where $\alpha(t)$ is a learning rate, an either monotonically decreasing or a constant function. In this work $\alpha=0.1$ for all measurements was used. It defines a fraction of the distance between $x_{i}$ and $v_{i}$ or how far the currently adapting vector $v_{i}$ would move towards the input vector $x_{i}$. The neighbourhood function $h_{c i}(t)$ defines the strength of adaptation for a node. This function has its maximal value for the best matching unit $c$, while the strength of adaptation for neighbouring units is decreasing according to their distance on the 2D-projection usually exponentially. A formula often chosen for the calculation of the neighbourhood function is a Gaussian shown in (4).

$$
h_{c i}=\exp \left(-\frac{\left\|r_{c}-r_{i}\right\|^{2}}{2 \sigma^{2}(t)}\right)
$$

Where the notion $\left\|r_{c}-r_{i}\right\|^{2}$ is a square of the geometric distance between the winner unit $c$ and unit $i, \sigma(t)$ is a monotonically decreasing function that covers a big part of the $2 \mathrm{D}$ space and decreases over time almost to the size of a single unit.

After the training procedure over some number of iterations, the mean quantization error $M Q E_{l}$ parameters of all nodes should be analysed. The higher $M Q E$ for a node is, the higher the density of the dissimilar input data mapped onto it. Thereby to represent the input vectors in that node in a more detailed way, new nodes have to be added to the grid. The unit $e$ with the highest $M Q E$ has to be found. Then the neighbouring unit which is most dissimilar to it, $d \in \mathcal{N}_{e}$, is to be determined using (5), where $\mathcal{N}_{e}$ denotes a set of the neighbouring units to the unit $e$.

$$
d=\arg \max _{i}\left(\left\|v_{e}-v_{i}\right\|\right), \quad v_{i} \in \mathcal{N}_{e}
$$

Depending on the position of the unit $d$, either a row or a column has to be added to the grid. The model vectors of new nodes are initialized as a mean between their neighbour units. The quantization error of the initial neuron can be calculated by (6).

$$
Q E_{0}=\sum_{x_{i} \in T} d\left(x_{i}, v_{j}\right)
$$

The GHSOM can be controlled by two parameters $\tau_{1}$ and $\tau_{2}$, where $\tau_{1}$ describes the minimum of the mean granularity of a map (7) allowing to set a condition about its size. The parameter $\tau_{1}$ is the horizontal growth factor of the map, while the parameter $\tau_{2}$ is the vertical growth factor of the map. The smaller the parameter $\tau_{1}$ is chosen, the bigger the map and the finer the projection of input vectors onto it will be. If a map $l$ has a mean quantization error $\left(M Q E_{l}\right)$ bigger than a part of the quantization error of its parent unit after some predefined number of iterations $\lambda$, then the map would grow horizontally until the condition (7) is satisfied, where $M Q E_{p}$ is the mean quantization error of a parent unit of the map. Instead of the mean values of all nodes in a map, the sum of the distances to their winning nodes could also be used. After the condition (7) is reached, the nodes which do not satisfy the quality criterion for a node described in (8) become an underlying map for better description of the input data.

$$
\begin{gathered}
M Q E_{l}<\tau_{1} \cdot M Q E_{p} \\
Q E_{i}<\tau_{2}
\end{gathered}
$$

\section{ALARM CONDITIONS 1 AND 2}

It was intended to use the nature of GHSOM to detect anomalies. As there are only two growth conditions in the GHSOM, one for vertical and one for horizontal growth, those two were used as alarm generating conditions. Therefore, by using the natural properties of the GHSOM it is possible to use two conditions only.

The following question was to be examined: If the GHSOM algorithm is applicable to form a normal model in the semi-supervised way during the training phase, would the map during the testing phase like in normal training grow, either horizontally or vertically or both? If so, it is believed that the test vector deviates too much from the trained model and it is regarded as anomaly because the normal model is assumed to be completely trained. The method is regarded as semi-supervised because all data has to be trained as "normal", and then all the other data are tested with the algorithm, i.e. test vectors.

During the training phase, Equation 7 is responsible for the vertical growth and Equation 8 for horizontal growth. During the testing phase the following steps are executed:

1) Recursively find the best matching unit (BMU) on the deepest possible layer.

2) Pretend that the testing vector is in the training phase, and if the map is supposed to grow, it means that the vector differs too much from the model, and it's marked as anomaly.

For a given test vector $t_{i}$ the best matching unit at the deepest possible layer has to be found first. $M Q E_{0}$ is the mean quantization error for the trained map which is calculated as shown in (9), where $m_{0}$ represents the mean of the input data and $n$ denotes the number of vectors projected onto the map. Then the $M Q E_{n e w}$ have to be calculated as moving average given in (10). The first alarm type is 
generated if the condition in (11) is fulfilled, which is referred to as condition 1 or first condition in the further text. If a true positive testing vector would be normally trained with the training vectors, then a horizontal growth of the map would be triggered, which means that the testing vector deviates from the data projected onto the map.

$$
\begin{gathered}
M Q E_{0}=\frac{1}{n} \cdot \sum_{x_{i} \in X}\left\|m_{o}-x_{i}\right\| \\
M Q E_{\text {new }}=M Q E_{l}+\frac{\left\|t_{i}-v_{c}\right\|-M Q E_{l}}{n+1} \\
M Q E_{\text {new }} \geq \tau_{1} \cdot M Q E_{0}
\end{gathered}
$$

The number of vectors that are mapped onto this unit is given by $n$, and $v_{c}$ is a multidimensional vector of the best matching unit. The condition described in (12) is referred to as condition 2 or second condition in the further text. The second alarm type is generated if the condition in (12) is fulfilled. The distance between trained vectors and their best matching units should always be smaller than the vertical growth factor of the map $\tau_{2}$. However, in the case when $\tau_{2}$ is chosen too small, the vertical growth condition can't be satisfied and after $\lambda$ iterations would be interrupted, which would result in a false alarm.

$$
Q E_{c} \geq \tau_{2}
$$

\section{EXPERIMENTAL SETUP}

The experiment should test whether the proposed detection method is able to recognize different attacks on confidentiality, availability and integrity aspects using the example of a PLC of the drilling module of a production line. The goal is to show that by using GHSOM it is possible to detect attacks on the industrial line with a high detection rate and a low false positive rate. The next goal is to show that the detection algorithm is able to detect attacks coming not only from unknown network participants but also from well known network participants that are being used as an attack platform.

Attacks on the production line. The following attacks were tested:

- Different types of TCP port scans (reconnaissance)

- PLC CPU sleep remote exploit (remote denial-of-service)

- Variable manipulation in the PLC

TCP port scanning is not a direct attack on a host but a tool to probe a host for open ports to identify running network services [5]. This information can be used for further exploitation of vulnerabilities of network services. For example if an attacker detects an open port 102, it can be assumed from a list of standard TCP ports for network services that the target is probably a Siemens PLC device. This information helps the attacker to determine possible attack platforms and remotely send an exploit to the target, e.g. the PLC CPU sleep remote exploit. This is an exploit sending a command over the S7COMM protocol to the PLC to go into sleep mode. This function is allowed for unauthorized hosts in the network. A typical procedure for sending a PLC stop command is shown on Figure 2. The third type of an attack is a variable manipulation. An unauthorized attacker can modify variables in the PLC by using the same

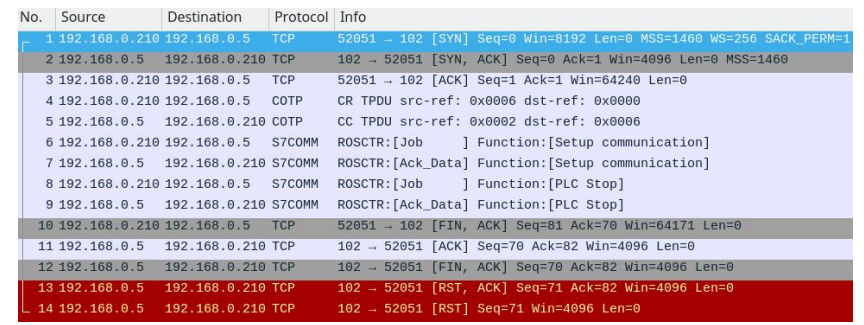

Figure 2: Sending stop command to Simatic S7-300 PLC over S7COMM protocol.

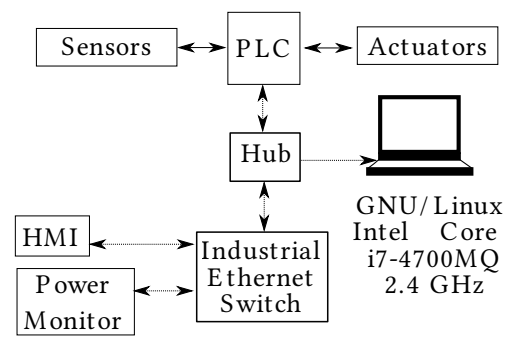

Figure 3: Communication flow inside a production module in the cyber-physical factory and the data capture setup.

S7COMM protocol which breaks the data integrity between the PLC and the touch panel. After that, the module of the production line can't be controlled from the touch panel anymore. The communication looks similar like depicted on Figure 2, except for the second "Job" block where the job is defined as a task to write a value into a given variable.

Testing platform. The testing platform represents a modular conveyor-driven production system that consists of five modules, each of them performing a manufacturing operation on a single production part [7]. Each of the modules is placed on a carrier pallet equipped with a radio-frequency identification (RFID) chip. All pallets carry a component part that is to be processed. The carrier pallets are transported on a conveyor belt. A programmable logic controller (PLC) carries out tasks of running production programs, reads electrical values of the module from a power monitoring device and their controlling, as well as the state of light-barrier sensors and air compression and identification of the pallet's carrier using RFID. The PLC communicates with the manufacturing execution system (MES) that is running on a single computer connected to the industrial Ethernet, and it also communicates with human-machine interfaces (HMI) in form of touch panels. The PLC Siemens S7-300 model is used in this factory. Communication between parts of a production module is depicted on Figure 3. The training was carried out on a GNU/Linux-driven laptop with an Intel Core i7-4700MQ CPU @ 2.40GHz on board.

Data acquisition and preprocessing. To get data from the PLC, an Ethernet hub was used. Ethernet frames going through the cable to and from the PLC were captured by the Wireshark program and saved as a Wireshark capture file. To derivate training and testing vectors, a Python script was used. From a predefined feature set 
it generated a text vector of features. Also it calculated $f_{\max }$ and $f_{\text {min }}$, the global maximum and minimum values respectively for every feature value $f_{i}$ in all datasets for further normalization of vectors. All elements of a vector were normalized to the interval $\hat{f}_{i} \in[0,1]$ using term (13).

$$
\hat{f}_{i}=\frac{f_{i}-f_{\min }}{f_{\max }-f_{\min }}
$$

Normalized vectors were placed in separate text files in commaseparated values (CSV) format. Those files were used for training a GHSOM ${ }^{1}$ and for testing accordingly. For all experiments the parameter $\lambda=50$ was chosen

Training data. The data were captured during the normal production process on a single PLC of a drilling module of the production line. Most of the network packets were based on the TCP protocol, namely $93.6 \%$ of all captured packets in the data set. Therefore, the packets used for the training set were based on TCP protocol only. Features for the training and test datasets from each Ethernet frame were used as follows: source MAC address, destination MAC address, IPv4 source address, IPv4 destination address, TCP source port, TCP destination port and only five of all possible TCP flags, namely: FIN, SYN, RST, PSH and ACK flags. Octets of MAC and IP addresses were separated as single features.

Feature selection. Start feature vectors were derived from a capture file of Wireshark. At the first step, each Ethernet frame was converted to text form. After that, all field values from Ethernet-, IPand TCP-headers were saved as comma-separated values. A time difference to the last captured packet was used as an additional feature that is not present in packet data directly. This information was read out from the capture file. Thereby the initial feature vectors were built up. Values for cyclic redundancy checks were omitted because of their almost uniform distribution. The total number of features resulted to be 43 . The feature selection depends on the type of attack. The types of attack that are expected to occur should be estimated. Concerning the 27 chosen features, the ones that are most likely expected to possibly be variable were chosen. As the port scans can use different source and destination IPs, TCP ports as well as TCP flags, those parameters were included in the feature set. For the ARP spoof attack, the MAC addresses are expected to differ from the legitimate ones, so they were included in the feature set. To check the importance of features, three feature selection techniques were used. If the feature selection would strictly follow just one of those techniques (instead of using the 27 chosen features), a different outcome concerning the receiver operating characteristic (ROC) and hence on the four parameters false positive, false negative, true positive and true negative can be expected. Following one of the selection techniques, it would also be possible to further reduce the amount of features, resulting likewise in a different ROC. However, if not enough features are chosen, the recognition performance of the classifier is likely to decrease. The feature selection algorithms for the classification problems using scikit-learn software were applied to estimate which features contribute the most to the classification prediction. The training set consists of 31,065 vectors. The testing set contains 2,443 vectors. Normal vectors were marked

\footnotetext{
${ }^{1}$ Implementation used from https://github.com/fffaraz/GHSOM-CPP and modified for intrusion detection purposes.
}

with 0 and the other vectors labelled as anomaly and marked with 1. After that, they were shuffled. Firstly univariate feature selection based on a $\chi^{2}$ statistical test was applied on the labelled dataset. ${ }^{2}$ The highest score was calculated for the sequence and acknowledgement numbers from the TCP header, TCP destination and source ports, TCP FIN, SYN, RST, PSH, ACK flags, Time-To-Live (TTL), last octet of the destination IP address, TCP window size and MAC addresses. The first three octets of the IP addresses received zero scores. The variance of them was zero because all participants are located within the same subnet 192.168.0/24 and no gateway communication took place. After that, a recursive feature elimination algorithm was applied on the dataset to select the 30 features that are contributing the most to the classifications. ${ }^{2}$ Eight of them are the bytes derived from MAC addresses and the remaining two are the last bytes from the source and destination IP address. The last test is the tree-based feature selection test with extra trees classifier. $^{2}$ The scores for features were different for every test run but always zero for 3 bytes of the subnet in source and destination IP addresses, IP version and header length field, differentiated services field, Ethernet type field, IP protocol field, and also for URG, ECE, CWR, TCP flags and urgent pointer field. The highest scores in this test were assigned to MAC addresses, last bytes of the source and destination IPs, TCP ports, FIN, SYN, RST, PSH, ACK flags. A weak feature importance was detected for elapsed time to the last packet and for the packet length. All three tests show zero influence of the first three bytes of the network IP address. To summarize the feature selection, the features that were chosen for the experiment do exhibit an importance in all three selection tests, except for the IP addresses, which were used completely because they may variate in an attack.

Testing data. For the detection rate evaluation different datasets were used, namely datasets containing:

1) 624 packets generated by connect scan,

2) 598 packets - only the SYN scan packets,

3) 594 packets - only the FIN scan packets,

4) 583 packets - only the NULL scan packets,

5) 14 packets of a remote exploit sent from the master computer forcing the PLC of a production line to go into sleep mode,

6) 15 packets sent from the master computer generated by a remote exploit forcing the conveyor to stop,

7) 15 packets sent from the master computer generated by a remote exploit forcing the conveyor to start.

Concerning datasets 1 to 4 , the IP-address doesn't appear during the training phase.

\section{EXPERIMENTAL RESULTS}

\subsection{Training dataset validation test}

To check how well the model is trained and to estimate the false positive rate, a training dataset validation test was carried out. To evaluate false positives, the source dataset was split into training and test datasets with 1:1 ratio. In the best case no training vectors should be detected as anomaly, because they all are extracted from the usual communication process of the factory and therefore should be true negative vectors. The results depicted on Figure 5

${ }^{2}$ http://scikit-learn.org/stable/modules/feature_selection.html 
show that the false positive rate depends non-linearly on values of $\tau_{1}, \tau_{2}$. The lowest total false positive rate is achieved at $\tau_{1}=\tau_{2}=0.1$, which results in rates of $f p_{\tau_{1}}=0.026 \%$ and $f p_{\tau_{2}}=0.003 \%$.

The amount and type of selected features also do have an impact on the false positive rate. By using all 43 features without feature selection instead of the 27 chosen features, using different hyperparameters $\tau_{1}, \tau_{2}$, the best achieved false positive rate has increased to $4.6 \%$. Also the training time has grown drastically. Those results show that feature engineering should be carried out carefully.

The ratio between training and evaluation datasets was 1:1 for a total of 31,065 testing vectors. It is worth to note that the training dataset should be held pure, because if any vectors derived from attacks would be trained, it will lead to undetected attacks

For the lowest false positive rate case all of the false positive vectors contained RST and ACK flags. At $\tau_{2} \geq 0.3$ the percentage of false positive vectors generated by the condition $\tau_{2}$ increases drastically. Using binary features such as TCP flags may cause those features to win a higher weighting in the distance calculation and in its turn can affect the best matching unit competition in the way that the wrong best fitting unit is chosen. An interesting fact is that condition 1 doesn't bring false positive rates higher than $0.1 \%$ to the model, even for higher values. At $\tau_{2}=0.2$ the 208 vectors that contained FIN ACK flags and one vector that included RST ACK flags simultaneously, and also seven vectors containing TCP keepalive packets were detected as anomalous. It can be interpreted that not enough keep-alive vectors were provided as input to avoid false positives in the training set. Above a certain threshold value the second proposed anomaly condition showed higher false positives rates. This can be interpreted in the way that the clustering of the data will not be optimal and more and more heterogeneous data are mapped onto a single node. Assuming that the map converges to a stable state, the vector weight values of the node after the training lie between the minimum and maximum value of the single vector weights that are mapped onto this node. If highly sparse data was mapped onto a single node, a higher standard deviation of the mapped data can be expected. Thereby the quantization error can exceed the $\tau_{2}$ parameter and generate a condition 2 alarm. To reduce the amount of false alarms and probably also decrease true positives, the mean quantization error of a node can be used.

\subsection{A trained GHSOM model topology}

An advantage of GHSOM is the possibility to graphically represent the data in a hierarchical structure and thereby check the similarity and dissimilarity of data. The map shows how many groups of data exist, which can be interesting for evaluation of large datasets like data communication patterns. Another possibility would be the graphical representation of the distance of testing vectors to map nodes, i.e. BMU. On Figure 4 the map trained with the parameters $\tau_{1}=\tau_{2}=0.1$ is depicted. From the graphical representation depicted on Figure 4, important information can be derived, e.g.:

(1) Similar data are placed together, for example data concerning TCP connection handling.

(2) Dissimilar data are placed in separate nodes, for example queries and responses.

(3) Communication between the energy monitor and the PLC (node with 1,258 vectors) received a hierarchical substructure

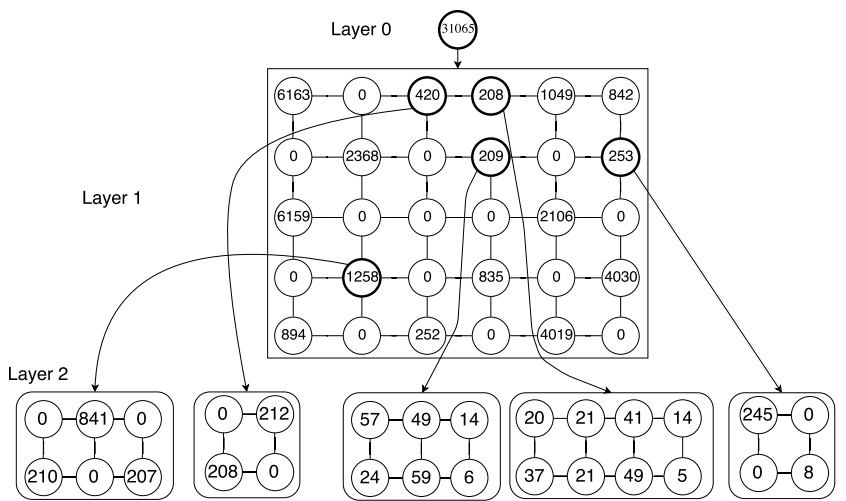

Figure 4: A trained GHSOM model with $\tau_{1}=\tau_{2}=0.1$

as follows: Three different nodes were created under layer 1 , one node each for connection establishment, ACK flags and connection termination.

In the following section the structure of the trained map is described. After the training, a map of $5 \times 6$ nodes was created. The numbers inside the nodes represent the number of training vectors mapped onto them. Five of these are marked with bold circles and exhibit underlying maps. The obtained map has only three layers including the zero layer. The biggest number of vectors mapped onto a single node is observed in a node containing 6,163 vectors, mostly consisting of ACK messages going from the PLC of the drilling module to a drilling machine control unit. Diagonally next to this node lies a node where 2,368 vectors were mapped onto. These are vectors going from the PLC to the drilling machine control unit too, but containing PSH ACK flags. Since the vectors are similar, these nodes should be topologically located nearby each other. Another node with 6,159 projected vectors consists of the vectors having PSH ACK flags going in the opposite direction or from the drilling control unit to the PLC. Vectors containing ACK flags are mapped only onto the node in the left bottom corner of the layer 1 of the map. A node with 1,258 mapped vectors contains a layer 2 map with three non-zero nodes. This map includes a node with 210 projected vectors containing SYN ACK flags going from the power monitor to the PLC, a node with 841 vectors containing ACK flags likewise in the mentioned direction between the same participants and a node with 207 mapped vectors exhibiting the mapping of FIN ACK flags. This node can be seen as a grown cluster containing vectors related to TCP connection handling for the power monitor device, which are split into three groups. A node with 420 mapped vectors has an underlying map with two effectively used nodes. The node with 208 projected vectors has connection termination flag RST going from the PLC to the power monitor device. Another node with 212 vectors contains the SYN flag, so it functions as a communication establishing cluster for PLC and the power monitor PAC3200. The MES system running on the master PC listens on two TCP ports, namely 2000 and 2001. 252 vectors coming from MES exhibited ACK messages from both open ports are mapped onto the node on the layer 1 in the bottom middle node. The queries to the MES coming from the PLC are mapped on the node containing 253 mapped vectors with an underlying map below it. In this map, 
245 vectors with PSH ACK flags directed to the destination TCP port 2001 were mapped on a single node, while 8 vectors that contained packets addressed to the TCP port 2000 were mapped onto a different node of this map. A node with 208 mapped vectors on the first layer map contains FIN ACK training vectors grouped by the TCP source port. The PLC establishes connection to the power monitor device, then queries the values, reads the response from the power monitor which contains the queried values. After that, the PLC closes the connection by sending the FIN ACK, RST ACK and RST sequence. The whole process is repeated with a period of about 1 second. Every time when a connection establishes a new incremented source, the TCP port number is used. A similar cluster with 209 mapped vectors contains vectors with RST ACK flags. A node with 1,049 projected vectors contains acknowledgement responses from the PLC to the power monitor. The power monitor uses the Modbus/TCP interface. After the connection has established, the PLC sends a query with PSH ACK flags for the energy values needed, and after that the PAC3200 sends a TCP ACK packet, followed by the response in a single packet with enabled PSH ACK flags. The queries from the controller are mapped onto a single node in the upper right node with 842 vectors. The responses from the power monitor are mapped onto a node containing 835 vectors. As one can see, the queries and responses are automatically topologically separated during the training process. The last participant in the communication with the PLC is a touch panel device. It communicates with the controller through a S7COMM protocol and establishes a continuous connection. The node with 2,106 mapped vectors containing the ACK flag consists of 2,019 vectors directed from the touch panel to the controller and 87 vectors pointing in the opposite direction. The mapping of differently natured vectors onto the same node can be explained as the similar distance to the best matching unit for both kind of vectors. The node with 4,018 projected vectors contains queries and the node with 4,030 consists of responses including TCP retransmission packets to them.

\subsection{TCP port scans}

For all of the data sets concerning attacks, namely TCP port scans, remote denial-of-service exploits and data manipulation, the true positive rate reached $100 \%$ when using the formula for condition 1 or the formula for condition 2, $\operatorname{cond}_{\tau_{1}} \vee \operatorname{cond}_{\tau_{2}}$. This means that every time an alarm for a vector was generated by one of the two conditions, it was treated as a true positive vector.

A TCP port scan is not a direct attack but could be used for preparation of it [5]. To detect a port scan, a rule-based approach could be applied, e.g. a stateful IDS. The port scan attack is used to test the detection of different attack scenarios. In the current work, the port scan is used evaluate the detection ability of the proposed method in anomaly detection mode. Therefore, this approach is more aimed at future attacks that aren't known yet, whilst rulebased approaches focus on the attacks described in the rules.

The test dataset consists of 624 true positive vectors that contain connection establishment attempts and responses to it to different ports of the production PLC from an IP address which is unseen in the training process. Not only the vectors generated by an attacker were assumed as true positives, but also a response from the legit PLC device being scanned was designated an undesired behaviour.
After the test with coefficient $\tau_{1} \leq 0.4$, the true positive rate for at least one of the two alarm conditions was $100 \%$. This means that every true positive vector was recognized either by the first or the second condition. Therefore, in those cases where the true positive rate of $100 \%$ is reached, the false negative rate, which is important for intrusion detection, is $0 \%$. It is worth noting that around $76 \%$ of the testing set vectors were marked as an anomaly for both of the alarm conditions. The $\tau_{1}$ coefficient for Equation 11 reached the highest true positive rate of $100 \%$ for a value of $0.2 \leq \tau_{1} \leq 0.4$, while the results worsened for increased values. The $\tau_{2}$ coefficient delivered the highest true positive rate of $100 \%$ for a value of $\tau_{2}=$ 0.1 . Therefore, using $\tau_{1}=0.1$ and $\tau_{2}=0.1$ allows to achieve the highest true positive rate and the lowest false positive rate.

The test dataset contains 598 true positive vectors related to the SYN scan of different ports of the production PLC from an IP address that doesn't appear during the training. The maximum true positive rate for the first detection condition was achieved by using $0.2 \leq \tau_{1}, \tau_{2} \leq 0.4$. Increasing $\tau_{1}$ didn't affect the detection rate by the first condition, while increasing $\tau_{2}$ reduced the detection rate almost to zero, detecting only two vectors containing SYN ACK flags sent during the scan process from the PLC to the attacker after an attempt to connect to the open ports 80 and 443 . The second alarm detection term showed its maximum for $\tau_{2}=0.1$.

Like the TCP SYN scan, for the FIN and NULL scans the two detection terms showed similar behaviour. For the FIN scan and for the NULL scan using the first detection condition the maximum of the true positive rate was achieved for both at $\tau_{1}=0.1$. The highest true positive alarm rate by the second condition for both FIN scan and NULL scan was achieved at $\tau_{2}=0.1$. At $\tau_{1}, \tau_{1} \geq 0.5$ the vectors marked as anomalous by the first condition haven't been detected by the second alarm term and vice versa: alarms generated by the second term haven't been detected by the first term.

\subsection{Remote denial-of-service exploits}

The evaluation of another test set containing vectors of remote CPU shutdown exploit started from a master computer was carried out. The data set contains a remote exploit consisting of 14 packets that establish connection to the PLC and send payload which turns the CPU of the PLC into sleep mode, followed by interruption of the production process at the attacked production module. This set represents the scenario when a network participant's device was infected with malware and used as an attack platform. Because many training vectors contain the master PC IP address and the exploitation TCP port, this dataset should depict the possibility to detect unusual behaviour in form of connection establishing coming from already known industrial network participants. It was intended to show that attacks coming from authorized participants could be detected via use of features such as IP, MAC addresses and TCP ports even without analysing the payloads of the network packets. For $\tau_{1}, \tau_{2}=0.1$, all true positive vectors could be detected. However, there are four vectors that were detected as anomaly, i.e. false positive, including the vector based on the exploit payload packet. Mostly vectors containing two TCP flags were detected as anomalous because of the larger distance to the best matching units and thereby higher $Q E_{c}$. A typical S7COMM transaction consists of 


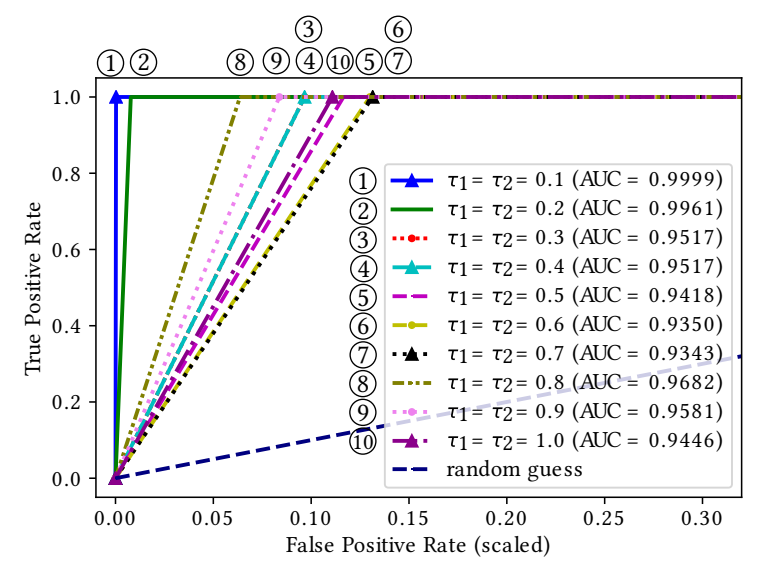

Figure 5: Receiver operating characteristics and area under the curve for resulting classifiers.

TCP connection establishing to port 102 of the PLC, connection oriented transport protocol (COTP) connection establishing, S7COMM setup communication, Job/AckData and TCP connection closing packets. The highest detection by Equation 11 was at $\tau_{1}=0.2$ with a true positive rate of $71 \%$.

\subsection{Data manipulation}

Two further kinds of attacks that were tested used the possibility of unauthorized variable modification access in the PLC. The first variable is responsible for the stop of the production cycle. The second one is responsible to set the line to an automatic mode which causes the conveyor to start and wait for an incoming pallet that carries a production part at a stopper. Both of the modifications lead to break data integrity between the PLC and touch panel causing the impossibility to control the production line from the touch panel. Both of the two datasets contain 15 vectors derived from the TCP packets of two separate attacks. Both attacks were started from the master PC assuming the scenario that the master PC was infected by malware, which makes it a platform for further attacks. The highest detection rate for the first condition was achieved at $\tau_{1}=0.2$.

Considering the false positive rates pictured at Figure 5, the lowest false positive in combination with the highest true positive results can be obtained by $\tau_{1}=\tau_{2}=0.1$.

\subsection{Training and testing time}

The testing time of all test datasets, which consisted of a total of 2,443 test vectors for different values of $\tau_{1}$ and $\tau_{2}$, is shown on Figure 6 . The lowest false positive and highest true positive rates are achieved at $\tau_{1}=\tau_{2}=0.1$ for the longest training time. A trade-off between training time and detection and error rates can be derived from Figure 6. After time-intensive GHSOM training, a fast detection is possible. The intrusion detection process of already trained models is taking place within a time span of tens of microseconds, including the alarm output. The testing of a model for $\tau_{1}=\tau_{2}=0.1$ with a total of 33,508 vectors took around 21 microseconds per vector in average, while the whole training process took over 200

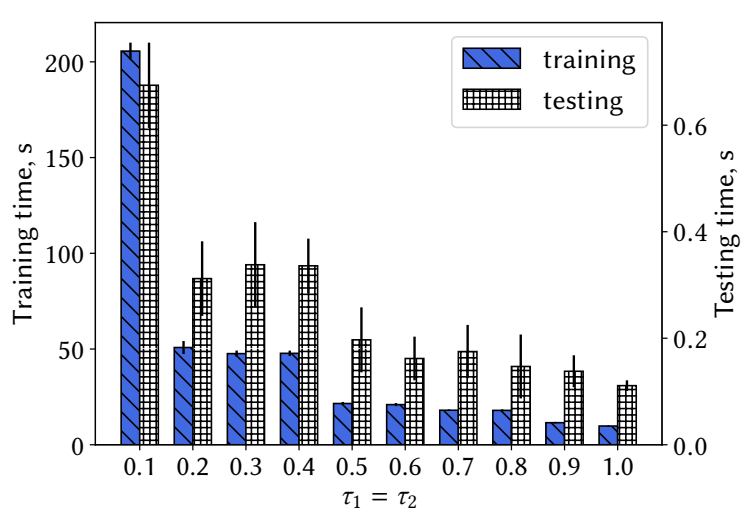

Figure 6: Comparison of training and testing time using different GHSOM parameters.

seconds. The anomaly detection algorithm proposed in this paper can provide a suitable approach that is fast enough for the use in industrial environments.

\section{COMPARISON WITH OTHER METHODS}

A GHSOM is one of several suitable approaches for data analysis in an IDS. The advantage of a GHSOM is its ability of semi-supervised learning, which is used for structuring data. In general, unsupervised or semi-supervised learning methods are preferred to supervised methods. This means there is no need to train the normal model with data derived from attacks. Periodical behaviour of industrial plants in the sense of deterministic network participants' behaviour can be mapped on the GHSOM, which means that the communication patterns are used to build a normal model of the data exchange between network participants. Because of the lack of data about attacks on industrial lines it is important to have big databases that contain information about the attacks themselves. GHSOM as a representative of semi-supervised learning is examined for the detection new, unprecedented attacks in the industrial environment. GHSOM may not be useful in an environment that generates highly variative data which will produce bigger models that increase the training time of a model and the model size itself.

For supervised learning such as support vector machines (SVM) or feed-forward neural networks (ANN), a set with known attacks has to be provided to build a proper model. The supervised techniques can provide better attack classification results and distinguish between the attack types. However, new attacks that were not trained in the detection model are not necessarily recognized. To compare different methods to GHSOM, the receiver operating characteristic of the proposed binary discrete classifier was contested with supervised and semi-supervised methods. The proposed approach has been tested in two different ways concerning the detection aspect:

(a) Training with all attack types in comparison to multi-class SVM and ANN (supervised methods), 
(b) Training without attacks that use S7COMM protocol in comparison with one-class SVM and local outlier factor methods (semi-supervised methods).

Experiment and results for variant (a). The whole dataset was shuffled and split into the datasets for training and testing. For both models the proportion between training and testing datasets was chosen to be 0.5 . The SVM model was trained using scikit-learn $\mathrm{v} 0.20 .2$ tool $^{3}$. The model parameters were set as default, but the kernel function was chosen to be a round base function $\exp \left(-\gamma\left\|x-x^{\prime}\right\|\right)$, which measures similarity between two inputs $x$ and $x^{\prime}$, where $\gamma=1 / n$ and $n$ is the number of features $(n=27)$ and the penalty parameter was left to be $C=1.0$. The ANN model was trained using Keras software ${ }^{4}$. The architecture of the network is built using two layers, where the first layer contained 4 nodes with rectifier activation function and a second layer consisting of a single node using a sigmoid activation function. The receiver operating characteristic of the proposed binary discrete classifier was compared with supervised methods based on SVM and ANN techniques.

For testing method (a), SVM and ANN have shown perfect classification ability with the AUC equal to 1.0 , as well as by GHSOM approach (AUC 0.9999). An important difference is that the proposed method using GHSOM has been trained only by using normal vectors without in advance providing information about possible future attacks (semi-supervised).

Experiment and results for variant $(b)$. Another test was carried out to check whether the novelty detection methods trained only on the normal vectors and TCP port scan vectors are able to detect other types of attacks which were started from the master computer. To make the task harder, most of the feature values that are present in untrained (to be tested) attack vectors are the same as in many normal traffic training vectors and the test should determine whether the trained models are able to detect the untrained attacks that look similar to the training data. The classifiers multiclass SVM and ANN with the same topology that was used in the test (a) have failed the test (b) completely, which means they are not applicable for the novelty detection. Concerning (b), only normal vectors were used as training dataset. A testing dataset a set with variable manipulation vectors and denial-of-service attacks mixed with normal vectors was used. It wasn't provided during the training phase. To compare the proposed method with other semi-supervised methods for novelty detection, two well-known approaches using scikit-learn were tested. The first one is the oneclass SVM model [22] and the second one is the local outlier factor (LOF) method in novelty detection mode [2]. The local outlier factor has shown perfect classification in the experiment (b). A slightly higher false positive rate resulted by the proposed GHSOM approach and yet a higher false alarm rate was shown by the SVM one-class model (see Figure 7).

Summary of the comparison of GHSOM with ANN, SVM, LOFmethods. For the used dataset from a real industrial line testbed the proposed anomaly detection method via GHSOM worked nearly as accurate as SVM and ANN for the case if the whole dataset was provided in advance, see test (a). It should be considered that the

\footnotetext{
${ }^{3} \mathrm{http://scikit-learn}$. org

${ }^{4}$ https://keras.io
}

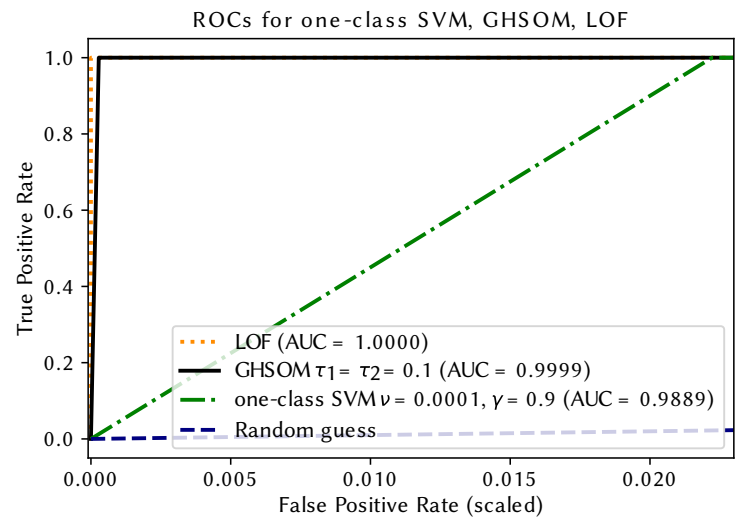

Figure 7: ROC for one-class SVM, GHSOM and LOF classifiers.

GHSOM lacks the need of complete supervision. For the case when some attack vectors were not provided in the training phase, the proposed method showed good classification results, whilst ANN and multi-class SVM using the same topology as in (a) couldn't distinguish any of the attacks they had not seen before. Because of that, a comparison with one-class SVM and local outlier factor method took place, see test (b). The proposed approach could distinguish between attack vectors and normal vectors slightly better (ROC AUC 0.9999) than the one-class SVM model (ROC AUC 0.9889), whereby the perfect novelty detection was shown by the LOF-method (ROC AUC 1.0). The possibility of mapping high-dimensional vectors onto $2 \mathrm{D}$ map can be used to graphically represent the hierarchical structure of the flowing data and concurrently be used to detect anomalies e.g. in intrusion detection tasks. The two tests have shown the applicability of the proposed method for the novelty detection problems such as anomaly detection in industrial networks.

The results of $100 \%$ accuracy of the classifiers indicate that the problem to distinguish anomalies for the studied industrial line was an easy task for machine learning if the attack vectors are given in advance but is not a feasible task for supervised learning if no attacks are known before. Therefore, the proposed approach via GHSOM can be used for research of future problems in more complicated tasks.

\section{CONCLUSION}

GHSOM is a semi-supervised algorithm allowing to train models without any knowledge about future attacks. Periodical behaviour of industrial plants allows to build a normal model of the communication of network participants. A deviation from the normal model can be seen as an anomaly to generate an alarm. So a typical novelty detection problem has to be solved. An intrusion detection method using GHSOM algorithm for building a normal communication pattern was proposed. Afterwards, the proposed method was evaluated with malicious vectors that were not provided in the training process. Those malicious vectors were derived from data of real attacks captured from the PLC of a real industrial line testbed while an attack on it took place. The attacks were covering 
confidentiality, integrity and availability aspects. The GHSOM was able to detect all of the true positive attack vectors with a ROC AUC of 0.9999 .

After the determination of the best parameters for GHSOM for classification, a comparison to multi-class SVM, ANN (supervised methods) and to one-class SVM and LOF (semi-supervised methods) took place. Two tests for trained supervised models were executed, one by training the model providing vectors of all attacks and another one evaluating the model without vectors derived from PLC protocol-specific attacks. For the first test, each of the supervised models has shown perfect classification. In the second test the multi-class SVM and ANN models have failed the test with the same layout used in the first test because they didn't detect attacks that were not trained before. The intrusion detection algorithms based on GHSOM and LOF showed nearly the same detection rates, while one-class SVM showed a little bit higher false alarm rate.

For supervised learning methods such as multi-class SVM or ANN, a set with known attacks should be provided to build a proper model. However, the efficiency of those supervised methods is strongly dependent on the degree of completeness of the used training dataset. The supervised techniques can provide better classification results and distinguish between the attack types. But information about the threats and future attacks is not always available. In those cases, the application of a semi-supervised or unsupervised method is necessary. Therefore, for industrial systems the sole use of supervised detection techniques is not sufficient, despite the good results at detection of known attack types, but the additional use of semi-supervised methods is reasonable. If a graphical evaluation of data is desired, then GHSOM is a good method to choose. In the present work the similarity and also the dissimilarity of data was checked via topological map. The proposed method using GHSOM can be used for offline and online intrusion detection likewise. In case of online detection, firstly an offline training of the GHSOM has to take place, followed by online detection.

\section{ACKNOWLEDGMENTS}

The authors thank Professor Felix Freiling from the Department of Computer Science, Friedrich-Alexander-Universität ErlangenNürnberg (FAU), for comments that greatly improved the work. The authors would also like to thank the anonymous referees for their valuable comments and helpful suggestions. This work is supported by the German Federal Ministry of Education and Research (Bundesministerium für Bildung und Forschung, BMBF) as a part of the SysKit project No.: 16KIS0661

\section{REFERENCES}

[1] Waseem Abbas, Aron Laszka, Yevgeniy Vorobeychik, and Xenofon Koutsoukos. 2015. Scheduling Intrusion Detection Systems in Resource-Bounded CyberPhysical Systems. In Proceedings of the First ACM Workshop on Cyber-Physical Systems-Security and/or PrivaCy (CPS-SPC '15). ACM, New York, NY, USA, 55-66. https://doi.org/10.1145/2808705.2808711

[2] Markus M. Breunig, Hans-Peter Kriegel, Raymond T. Ng, and Jörg Sander. 2000. LOF: Identifying Density-based Local Outliers. SIGMOD Rec. 29, 2 (May 2000), 93-104. https://doi.org/10.1145/335191.335388

[3] Varun Chandola, Arindam Banerjee, and Vipin Kumar. 2009. Anomaly Detection: A Survey. Comput. Surveys 41, 3, Article 15 (July 2009), 58 pages. https://doi.org/ $10.1145 / 1541880.1541882$

[4] Kruti Choksi, Bhavin Shah, and Ompriya Kale. 2014. Intrusion Detection System using Self Organizing Map: A Survey. In Neural networks: the official journal of the International Neural Network Society, Vol. 4. 11-16.
[5] Marco de Vivo, Eddy Carrasco, Germinal Isern, and Gabriela O. de Vivo. 1999. A Review of Port Scanning Techniques. ACM SIGCOMM Computer Communication Review 29, 2 (April 1999), 41-48. https://doi.org/10.1145/505733.505737

[6] Ashfaq Hussain Farooqi and Farrukh Aslam Khan. 2012. A Survey of Intrusion Detection Systems for Wireless Sensor Networks. International fournal of Ad Hoc and Ubiquitous Computing 9, 2 (Feb. 2012), 69-83. https://doi.org/10.1504/ IJAHUC.2012.045549

[7] Festo. 2019. CP Factory - The Cyber-Physical Factory. (2019). https://www.festo-didactic.com/int-en/learning-systems/learning-factories, cim-fms-systems/cp-factory/cp-factory-the-cyber-physical-factory.htm

[8] Hamid Reza Ghaeini, Daniele Antonioli, Ferdinand Brasser, Ahmad-Reza Sadeghi, and Nils Ole Tippenhauer. 2018. State-aware Anomaly Detection for Industrial Control Systems. In Proceedings of the 33rd Annual ACM Symposium on Applied Computing (SAC '18). ACM, New York, NY, USA, 1620-1628. https://doi.org/10. $1145 / 3167132.3167305$

[9] Hamid Reza Ghaeini and Nils Ole Tippenhauer. 2016. HAMIDS: Hierarchical Monitoring Intrusion Detection System for Industrial Control Systems. In Proceedings of the 2Nd ACM Workshop on Cyber-Physical Systems Security and Privacy (CPS-SPC '16). ACM, New York, NY, USA, 103-111. https: //doi.org/10.1145/2994487.2994492

[10] Manasi Gyanchandani, JL Rana, and RN Yadav. 2012. Taxonomy of anomaly based intrusion detection system: a review. International fournal of Scientific and Research Publications 2, 12 (2012), 1-13.

[11] Dina Hadžiosmanović, Robin Sommer, Emmanuele Zambon, and Pieter H. Hartel. 2014. Through the Eye of the PLC: Semantic Security Monitoring for Industrial Processes. In Proceedings of the 30th Annual Computer Security Applications Conference (ACSAC '14). ACM, New York, NY, USA, 126-135. https://doi.org/10.1145/2664243.2664277

[12] Jun Inoue, Yoriyuki Yamagata, Yuqi Chen, Christopher M. Poskitt, and Jun Sun. 2017. Anomaly Detection for a Water Treatment System Using Unsupervised Machine Learning. 2017 IEEE International Conference on Data Mining Workshops (ICDMW) (Nov 2017). https://doi.org/10.1109/icdmw.2017.149

[13] Dennis Ippoliti and Xiaobo Zhou. 2010. An Adaptive Growing Hierarchical Self Organizing Map for Network Intrusion Detection. In 2010 Proceedings of 19th International Conference on Computer Communications and Networks. 1-7. https://doi.org/10.1109/ICCCN.2010.5560165

[14] iTrust and Singapore University of Technology \& Design. 2019. Secure Water Treatment - iTrust. (2019). https://itrust.sutd.edu.sg/research/testbeds/ secure-water-treatment-swat/

[15] William Jardine, Sylvain Frey, Benjamin Green, and Awais Rashid. 2016. Senami: Selective non-invasive active monitoring for ics intrusion detection. In Proceedings of the 2nd ACM Workshop on Cyber-Physical Systems Security and Privacy. 23-34. https://doi.org/10.1145/2994487.2994496

[16] Moshe Kravchik and Asaf Shabtai. 2018. Detecting Cyber Attacks in Industrial Control Systems Using Convolutional Neural Networks. In Proceedings of the 2018 Workshop on Cyber-Physical Systems Security and PrivaCy (CPS-SPC '18). ACM, New York, NY, USA, 72-83. https://doi.org/10.1145/3264888.3264896

[17] Robert Mitchell and Ing-Ray Chen. 2014. Review: A Survey of Intrusion Detection in Wireless Network Applications. Computer Communications 42 (April 2014), 1-23. https://doi.org/10.1016/j.comcom.2014.01.012

[18] Andres Ortiz, Julio Ortega, Alberto Prieto, and Antonio F Díaz. 2011. Improving network intrusion detection with growing hierarchical self-organizing maps. In Proceedings on the International Conference on Artificial Intelligence (ICAI). The Steering Committee of The World Congress in Computer Science, Computer Engineering and Applied Computing (WorldComp), 1.

[19] Esteban J. Palomo, Enrique Domínguez, Rafael Marcos Luque, and José Muñoz. 2009. An Intrusion Detection System Based on Hierarchical Self-Organization. In Proceedings of the International Workshop on Computational Intelligence in Security for Information Systems CISIS'08. Springer, Berlin, Heidelberg, 139-146. https://doi.org/10.1007/978-3-540-88181-0_18

[20] Andreas Rauber, Dieter Merkl, and Michael Dittenbach. 2002. The growing hierarchical self-organizing map: exploratory analysis of high-dimensional data. IEEE transactions on neural networks / a publication of the IEEE Neural Networks Council 13, 6 (2002), 1331-41. https://doi.org/10.1109/TNN.2002.804221

[21] Juan Enrique Rubio, Cristina Alcaraz, Rodrigo Roman, and Javier Lopez. 2017. Analysis of Intrusion Detection Systems in Industrial Ecosystems. In Proceedings of the 14th International foint Conference on e-Business and Telecommunications Volume 6: SECRYPT, (ICETE 2017). INSTICC, SciTePress, 116-128. https://doi.org/ $10.5220 / 0006426301160128$

[22] Bernhard Schölkopf, John C. Platt, John C. Shawe-Taylor, Alex J. Smola, and Robert C. Williamson. 2001. Estimating the Support of a High-Dimensional Distribution. Neural Computation 13, 7 (July 2001), 1443-1471. https://doi.org/ 10.1162/089976601750264965

[23] Yaping Zhang, Wenxiu Bu, Chang Su, Luyao Wang, and Han Xu. 2016. Intrusion detection method based on improved growing hierarchical self-organizing map. Transactions of Tianjin University 22, 4 (01 Aug 2016), 334-338. https://doi.org/ 10.1007/s12209-016-2737-4 